\title{
Miti e utopie della modernità nelle Riflessioni sulla violenza di Georges Sorel
}

\author{
di Viviana Galletta \\ vivianagalletta@hotmail.com
}

\begin{abstract}
This paper analyses the work Riflessioni sulla violenza written by Georges Sorel and published in 1908. The principal aim of this paper is to present the deep relationship between myth, violence and politics in order to reevaluate how irrational forces have guided social movements and revolutions. The distinction between the notions of force and violence introduces the central thesis of Georges Sorel's political thought, which is called anarcho-syndacalism. More specifically, George Sorel puts together Marx and Bergson in order to develop a severe criticism of the Third Republic and to theorize the role of violence in the transition from capitalism to socialism. Through the myth of the general strike, Sorel introduces his philosophical perspective on social struggles against the parliamentarism.
\end{abstract}

Keywords: Violence, Myth, General Strike, Utopia

\section{Introduzione}

La riflessione politica di Georges Sorel $^{1}$ fa perno, in verità non troppo sorprendentemente - almeno per chi è avvezzo alla ricorrente intersezione tra riflessione politica e riflessione sul mito -, sulle nozioni di mito e di utopia. Tale prospettiva teorica presenta, nei termini di un'antitesi concepita sul piano ideologico e simbolico, le due diverse direzioni assunte dal movimento storico-politico nella Francia della Terza Repubblica: il sindacalismo

\footnotetext{
${ }^{1}$ Per una ricognizione bibliografica sull'autore - sebbene non adeguatamente aggiornata - si veda S. Sand, "Bibliographie des études sur Georges Sorel", Mil neuf cent. Revue d'histoire intellectuelle, I, 1983, pp. 173-206. Una più recente nota bibliografica è consultabile all'interno dell'edizione italiana degli scritti politici di Sorel: G. Sorel, Scritti politici, a cura di R. Vivarelli, UTET, Milano, 2006, pp. 55-78. Si segnala, inoltre, la seguente opera monografica di taglio biografico: M. Gervasoni, Georges Sorel, una biografia intellettuale. Socialismo e liberalismo nella Francia della Belle époque, Unicopli, Milano, 1997.
} 
rivoluzionario e la politica della rappresentanza parlamentare abbracciata dai partiti.

Il tema della scissione nella lotta tra le classi sociali, attuabile per mezzo del mito dello sciopero generale, e, all'opposto, quello della tenuta dell'unità sociale per mezzo del parlamentarismo costituiscono, negli scritti politici soreliani, il nodo cruciale intorno a cui si sviluppa una severa critica alla politica e alla morale della modernità nella direzione di quella che l'autore ritiene essere l'unica possibile attualizzazione dello spirito marxiano: l'immediata e violenta irruzione del mito dello sciopero generale in un presente pacificato dalle sedute conciliatorie della politica parlamentare ${ }^{2}$. Nella definizione soreliana, il mito sociale dello sciopero generale configura «una organizzazione di immagini capaci di evocare istintivamente tutti i sentimenti che corrispondono alle diverse manifestazioni della guerra intrapresa dal socialismo contro la società moderna» ${ }^{3}$.

Questo contributo si sofferma ad analizzare, nello specifico, lo scritto Riflessioni sulla violenza $a^{4}$ con lo scopo di documentare il nesso tra mito, violenza e azione politica nella riflessione soreliana e di mettere in luce, dunque, il potenziale sovversivo contenuto nella dimensione del simbolico.

\section{La Francia della Terza Repubblica: assetto statale e antistatalismo soreliano}

All'indomani della battaglia di Sedan il crollo dell'impero di Napoleone III lascia spazio all'instaurarsi di un nuovo assetto politico che si manterrà sostanzialmente fino al 1940, ovverosia la Terza Repubblica francese. I motivi

\footnotetext{
${ }^{2}$ G. Sorel, Riflessioni sulla violenza, in G. Sorel, Scritti politici, cit., p. 147.

${ }^{3}$ Ivi, p. 219.

${ }^{4}$ Le Réflexions sur la violence apparvero nel 1906 in Le Mouvement socialiste, XIX, Paris. Nella rivista diretta da Lagardelle Sorel riprendeva, pur con modifiche ed ampliamenti, alcuni articoli pubblicati in Italia nel Divenire Sociale di Enrico Leone, poi riuniti in un volume dal titolo Lo sciopero generale e la violenza. La versione definitiva delle Réflexions fu pubblicata nel 1908 in Pages libres. Nelle edizioni seguenti (del 1912 e del 1919) Sorel aggiunse tre appendici (Unité et multiplicité; Apologie de la violence; Pour Lénine).
} 
più ricorrenti della riflessione politica soreliana, a cominciare dalla contrapposizione tra una morale individualistica e una morale pubblica ${ }^{5}$, rimontano all'osservazione di tale assetto politico-sociale.

L'analisi soreliana sconfina nella radicale contestazione dello Stato moderno in quanto ritenuto espressione di logiche utilitaristiche e, per questa ragione, immorali 6 :

\begin{abstract}
Il Sorel ha fatto segno alle sue invettive e ai suoi sarcasmi lo Stato moderno, o per essere più esatti la Terza Repubblica francese. [...] In sostanza quel ch'egli rimprovera a questo Stato moderno non era di essere troppo Stato, ma di non esserlo abbastanza: soltanto il "troppo" e l'“abbastanza" non vanno presi materialmente, quantitativamente, ma in senso ideale e morale. Il moltiplicarsi delle ingerenze statali ch'egli abbominava nello Stato moderno significava per lui un assoggettamento dello Stato agli interessi particolari, al servizio degli individui, e cioè della borghesia avida di facili arricchimenti, degli intellettuali procaccianti e parassiti?
\end{abstract}

Dunque, lo Stato moderno - nella forma della Terza Repubblica francese avrebbe sostituito ad una moralità pubblica il tornaconto individuale e gli interessi particolari delle forze politiche di volta in volta al potere. Persino $\mathrm{i}$ socialisti, divenuti politicanti, indirizzano i movimenti delle masse ad uno scopo politico, convertendo gli istinti di rivolta in forza elettorale ${ }^{8}$.

L'adesione di Georges Sorel al sindacalismo rivoluzionario ${ }^{9}$, maturata negli anni dell'affare Dreyfus e dell'esperienza fallimentare - almeno secondo il suo

\footnotetext{
${ }^{5}$ Sull'accusa di individualismo rivolta alla moderna democrazia della Terza Repubblica francese, le cui logiche avrebbero corrotto le antiche virtù civili e morali, si veda R. Vivarelli, Introduzione, in G. Sorel, Scritti politici, cit., pp. 13-15.

${ }^{6}$ Sulla critica alla Terza Repubblica si veda M. Curtis, Three Against the Third Republic, Sorel, Barrès, and Maurras, Princeton University Press, Princeton 1959.

7 S. Salvatorelli, "Profilo di Georges Sorel", Pegaso, II, 1930, p. 36.

${ }^{8}$ Cfr., G. Sorel, Riflessioni sulla violenza, cit., p. 251.

9 Sulla delusione politica di Sorel nei riguardi di un possibile mutamento interno alla democrazia e sul suo conseguente passaggio dal riformismo al rivoluzionarismo cfr. H. S. Hughes, Consciousness and society: the reorientation of European social thought, 1890-1930, Knopf, New York 1958, pp. 91-92: «Initially, in terms of Sorel's own biography, it seems clear that his shift from revisionism to the advocacy of violence reflected the enormous disillusionment with the democratic process. Like so many other French intellectuals, he had been a militant Dreyfusard, and his revisionist position of the late 1890's logically paralleled the "optimistic" phase of unity between Socialists and middle-class democrats that the great affaire had inspired». Per un approfondimento della posizione revisionista di Sorel, databile
} 
giudizio - di un'azione socialista interna alle dinamiche parlamentari, trova una traduzione, sul piano della concettualizzazione filosofica, nelle Riflessioni sulla violenza. L'esperienza politica e intellettuale di Georges Sorel - in particolare la meditazione sui testi di Marx e di Bergson - fa capolino nell'elaborazione di una riflessione antistatalista imperniata sul mito dello sciopero generale.

L'antistatalismo soreliano matura, dunque, negli anni in cui lo Stato francese, nella forma della Terza Repubblica, andava rafforzandosi in quanto dispositivo neutro di potere agognato da tutte le forze politiche, socialisti compresi. Proprio nell'avvicendamento alla detenzione della forza pubblica Sorel individua il carattere dominante delle dinamiche politiche del XIX secolo, connotate, a detta dell'autore francese, dalla soppressione di ogni tentativo di rinnovamento morale a vantaggio dello Stato e del suo consolidamento: «Ma combattendo in tal modo i detentori della forza pubblica, non desiderano affatto che questa venga soppressa; poiché è loro desiderio di utilizzarla un bel giorno a loro profitto; tutte le perturbazioni rivoluzionarie del XIX secolo si sono concluse con un rafforzamento dello Stato» ${ }^{10}$.

All'opposto delle dinamiche parlamentari, sostanzialmente incentrate sull'azione di mediazione delle istanze sociali svolta dai partiti politici, il sindacalismo rivoluzionario promuoveva il sindacato come un canale di azione diretta e spontanea delle masse operaie, rifiutando ogni tentativo di incanalare i conflitti sociali all'interno delle istituzioni statali11.

negli anni che vanno dal 1897 al 1900, si veda P. Ferrario, "Sorel e la critica al marxismo", Il Politico, XLVIII/3, 1983, pp. 557-574.

10 G. Sorel, Riflessioni sulla violenza, cit., p. 101.

11 Sulla funzione antistatale del sindacalismo rivoluzionario si veda P. Ferrario, "Sorel e il mito della violenza (Per una rilettura delle Réflexions)", Il Politico, LI/1, 1986, pp. 63-81: «[...] le speranze riformiste, suscitate negli ambienti socialisti moderati dalla entrata di Millerand nel governo Waldeck-Rousseau, erano state completamente deluse. Grandi scioperi avevano opposto violentemente gli operai alle truppe militari, facendo tramontare ogni speranza di pace sociale. In quegli anni il sindacalismo sviluppò rapidamente la propria azione tra le masse proletarie: il rifiuto di ogni accordo politico con forze parlamentari stava a significare il rifiuto completo dell'autorità statale; in una parola la negazione dello Stato» (p. 71). 
L'elaborazione di una prospettiva antistatalista - nella forma dell'anarcosindacalismo - si incentra, dunque, sulla contestazione dello Stato moderno, alla luce delle vicende politiche di cui Sorel era acuto testimone negli anni del governo Waldeck-Rousseau, sostenuto da radicali e socialisti.

L'antitesi tra le nozioni di forza e di violenza gioca in tal senso un ruolo centrale: «[...] diremo dunque che la forza ha per oggetto di imporre la organizzazione di un certo ordine sociale nel quale governa una minoranza, mentre la violenza tende alla distruzione di questo ordine» ${ }^{12}$.

La lotta per la conquista della forza rappresenta una dinamica precipuamente borghese e intra-statale, e cioè propria della politica parlamentare dei partiti, a cui i socialisti si sarebbero incautamente adeguati. La forza statale rappresenta, difatti, un'istanza di pacificazione e di elusione di ogni conflitto poiché accomuna le diverse istanze politico-sociali nella medesima con-fusione utilitaristica ed immorale realizzata nello Stato borghese.

Al contrario, la violenza proletaria, che si esprime nello sciopero generale, esaspera la scissione tra le parti sociali, realizzando l'unico autentico atto politico - che possa davvero dirsi pubblico e, dunque, morale ${ }^{13}$ - perché realmente conflittuale rispetto alle contraddizioni del presente. Tali contraddizioni sono sostanzialmente quelle espresse dai socialisti ufficiali i quali, in luogo della libertà del produttore, promuovono una disciplina nell'officina, ovverosia una riconferma della condizione dei lavoratori come

12 G. Sorel, Riflessioni sulla violenza, cit., p. 276.

13 Ivi, pp. 346-348: «[...] noi abbiamo una nuova ragione per credere nell'avvento del socialismo, poiché esso rappresenta il più alto ideale morale che l'uomo abbia mai concepito. Non si tratta di una religione nuova, che matura sotterra, senza l'aiuto dei pensatori borghesi; è una virtù che nasce, una virtù che gli intellettuali della borghesia sono incapaci di comprendere, una virtù che può salvare la civiltà - e Renan sperava che sarebbe salvata ma con l'eliminazione totale di quella classe alla quale Renan aveva appartenuto. [...] Il sublime è morto nella borghesia ed essa è dunque condannata a non avere più morale». Nella prospettiva soreliana, quindi, la borghesia e con essa i politicanti socialisti sarebbero privi dello slancio ideale in grado di rispondere al problema più urgente del tempo presente, ovverosia quello di concepire il passaggio dall'uomo attuale allo stato di produttore libero privo di padroni (vale a dire il passaggio dal capitalismo al socialismo). 
strumenti passivi al servizio degli interessi particolari delle minoranze che si avvicendano al potere ${ }^{14}$.

Il fenomeno della violenza proletaria nella forma dello sciopero generale costituisce dunque, nell'ottica soreliana, la piena applicazione della critica marxiana allo Stato contro le derive del marxismo contemporaneo: «La violenza proletaria muta l'aspetto di tutti i conflitti nel corso dei quali è dato osservarla; poiché essa nega la forza organizzata della borghesia e pretende sopprimere lo Stato che ne forma il nodo centrale. [...] è perciò che i nostri socialisti parlamentari, che sono i figliolini della borghesia e che niente sanno all'infuori della ideologia dello Stato, sono tutti disorientati allorché si trovano in presenza della violenza proletaria» ${ }^{15}$.

Se l'adesione allo spirito marxiano mette in gioco la critica allo Stato, concepito come garante delle contraddizioni del capitalismo borghese, la lettura di Bergson suggerisce a Sorel la teoria del mito come via per comprendere intuitivamente l'essenza del socialismo: «Le mythe de la grève générale nous permet donc de saisir tout le socialisme intuitivement; aucune analyse rationaliste ne permettra jamais d'appréhender l'essence du socialisme aussi bien que par le mythe de la grève générale, c'est-à-dire par cette intuition non scientifique à laquelle Bergson demande de nous découvrir la réalité de l'être» ${ }^{16}$.

La riabilitazione dell'elemento irrazionale del mito, inteso come forza storica capace di mobilitare le masse e di produrre un'azione sociale nell'immediatezza del tempo presente, confligge con l'intellettualismo borghese, dedito a mantenere lo stato delle cose per mezzo della celebrazione retorica del passato o della presentazione utopica del futuro. Nella definizione soreliana, il mito acquisisce un carattere marcatamente morale in quanto

\footnotetext{
14 Ivi, p. 357.

15 Ivi, p. 102.

16 P. Andreu, "Bergson et Sorel", Les études bergsoniennes, vol. III, Albin Michel, Paris, 1952, p. 70 .
} 
complesso di immagini capace di muovere l'azione delle masse operaie nella direzione di una «rivoluzione assoluta»17: "Il mito come costruzione irrazionale che permette alle masse (alle quali si rivolge) di entrare nel "regno della libertà”, sottraendosi ad ogni condizionamento sociale, il mito, insomma, come integrale evasione sublime, al di fuori di ogni considerazione e di ogni valutazione pratica, ed insindacabile anche sul piano dell'efficacia storica, può ricevere solamente un'adesione morale e non intellettualisticamente mediata» ${ }^{18}$.

La filosofia bergsoniana e, nello specifico, il Saggio sui dati immediati della coscienza ${ }^{19}$ prestano il fianco ad approfondire la teoria dei miti che fa da sostrato all'azione proletaria e al sindacalismo soreliano. I temi dell'immediatezza e del movimento creatore, intesi come momenti rari di libertà del soggetto rispetto all'azione politica, mettono sotto accusa il sistema dei partiti, retto sulle logiche della mediazione e della rappresentanza, promuovendo, in luogo di un riformismo graduale da collocarsi nel tempo spazializzato, il puro movimento rivoluzionario che si posiziona nella durata senza soste né interruzioni. Evidentemente, Sorel sfrutta la critica bergsoniana alla spazializzazione del tempo per impostare la sua analisi delle dinamiche parlamentari. Nell'introduzione alle Riflessioni, la contrapposizione tra il parlamentarismo e il sindacalismo rivoluzionario viene infatti presentata alla luce dei due Io diversi bergsoniani, di cui l'uno configura la proiezione esterna e spazializzata dell'altro. L'agire libero e spontaneo delle masse operaie nel contesto dello sciopero generale si posiziona, al pari dell'io profondo bergsoniano, nella pura durata e, cioè, nella coscienza creatrice capace di «spezzare i quadri storici» 20 .

${ }_{17}$ Cfr. G. Sorel, Riflessioni sulla violenza, cit., p. 109.

${ }_{18}$ P. Ferrario, "Sorel e il mito della violenza (Per una rilettura delle Réflexions)", cit., p. 13.

${ }^{19}$ H. Bergson, Essai sur les données immédiates de la conscience, Félix Alcan, Paris 1889; Saggio sui dati immediati della coscienza, tr. it. di G. Bartoli, Bollati Boringhieri, Torino 1964; tr. it. di F. Sossi, Raffaello Cortina Editore, Milano 2002.

${ }^{20}$ Cfr. G. Sorel, Riflessioni sulla violenza, cit., pp. 109-112. 
La «trasformazione assoluta e irrevocabile» 21 nella direzione di un rinnovamento sociale, attuabile dalla forza irrazionale del mito dello sciopero generale, contempla l'istituzione di un rapporto diretto con i fatti del presente. Il mito, a differenza dell'attitudine intellettualista, consente la realizzazione del passaggio dai principi all'azione:

\begin{abstract}
Sorel's interest in myth arose from his belief that "intellectualist philosophy" could not explain why a man would willingly sacrifice his life for an ideal. How could one account for revolutions or empires without positing some superior motive force acting within people? In a more general sense the passage from principles to action always contained the presence of myth, which Sorel considered to be a group of images intuitively or viscerally apprehended. [...] While most human activity proceeded from the calculation of self-interest and evolved from daily routines, myths gripped the mind with a much greater tenacity than self-interest or habit and enabled people to act in radically new ways. [...] Sorel hoped that a contemporary myth like that of a general strike might bridge the growing gulf he perceived between thought and action in European socialism ${ }^{22}$.
\end{abstract}

Il ragionamento soreliano intorno alla funzione del mito, inteso come immagine capace di acuire i conflitti e di sostenere l'azione delle masse nella violenta scissione operata dallo sciopero generale, introduce una netta presa di distanza dal socialismo riformista. In tal senso, Sorel presenta il mito come un dispositivo assolutamente altro dall'utopia, intesa come strumento ideologico proprio del socialismo riformista.

L'utopia costituisce, infatti, un'astrazione dal fatto immediato e, in quanto prodotto di un lavoro intellettuale e teorico teso a stabilire un modello con il quale confrontare la società esistente e risolverne le problematiche, orienta l'azione di riforma del sistema collocandola in una traiettoria di approssimazione graduale al modello ipotizzato: «Les utopistes sont tous, plus ou moins, intellectualistes; tous suppriment, d'une manière plus ou moins apparente, les sentiments dans la vie sociale» ${ }^{23}$. Il mito, al contrario,

\footnotetext{
${ }^{21}$ Ivi, p. 262.

${ }^{22}$ M. Tager, "Myth and Politics in the Works of Sorel and Barthes", Journal of the History of Ideas, XLVII, 1986, p. 626.

${ }^{23}$ G. Sorel, "Y a-t-il de l'utopie dans le marxisme?", Revue de métaphysique et de morale, II, $7^{\text {e }}$ année, 1899, 152-175, qui p. 171.
} 
interviene nel presente come sentimento capace di azionare le masse senza incanalarle nei ragionamenti e nelle astrazioni dei teorici.

Quella proposta da Sorel nelle Riflessioni si presenta, dunque, come una forma inedita di antistatalismo, sorta dalla volontà di completare - e non semplicemente commentare - la dottrina di Marx alla luce delle novità sociali del tempo presente ${ }^{24}$, facendo del bergsonismo un elemento fondativo del sindacalismo. In sintesi, Sorel introduce l'elemento anarchico nel sindacalismo, promuovendolo come alternativa rispetto alle deviazioni del socialismo riformista verso concezioni borghesi.

\section{Violenza e moralità: il mito dello sciopero generale}

Quella elaborata da Georges Sorel nelle Riflessioni sulla violenza è una prospettiva filosofica atta a ristabilire il valore morale della violenza nel contesto della lotta tra classi sociali, alla luce della deriva parlamentarista del socialismo. Va detto che la tesi soreliana circa la moralità della violenza è debitrice dell'insegnamento nietzschiano, con specifico riguardo alla nota opposizione tra due gruppi di valori morali. L'angolazione soreliana, tuttavia, introduce un'originale considerazione circa i valori implicati nei rapporti civili. Nello specifico, la morale dei produttori gli consente di articolare ulteriormente la distinzione tra il sindacalismo rivoluzionario e il socialismo

\footnotetext{
${ }^{24}$ È lo stesso Sorel ad indicare la volontà di guardare all'insegnamento di Marx nell'ottica di una sua attualizzazione coerente con il tempo presente e con i nuovi fatti sociali (nello specifico, con la pratica dello sciopero generale): «Disgraziatamente Marx non aveva sotto gli occhi i fatti che ci sono divenuti familiari: noi sappiamo meglio di lui ciò che sono gli scioperi [...] in fatto di violenza noi abbiamo delle idee che Marx non avrebbe potuto formarsi facilmente; noi dunque possiamo completare la sua dottrina, invece di commentare i suoi testi come per tanto tempo hanno fatto dei malfortunati discepoli»(G. Sorel, Riflessioni sulla violenza, cit., pp. 115-116).

Dunque, completare la dottrina di Marx significa, per Sorel, aggiornarla alla luce delle novità del tempo presente (si pensi alla novità della distinzione tra forza borghese e violenza proletaria) ed è su tale impostazione che egli concepisce la missione della cosiddetta nuova scuola: «Non è diluendo le frasi di Marx in verbosi commentari che si può mantenere intatta l'idea rivoluzionaria; è adattando sempre il pensiero a quei fatti che possono prendere un aspetto rivoluzionario. Solo lo sciopero generale, oggi, può produrre tale risultato» (Ivi, pp. 329-330).
} 
di Stato, indicando alle masse operaie la via per rivendicare la propria autonomia nella prospettiva di un «radicale rovesciamento della condizione servile» ${ }^{25}$. Insomma, per Sorel si tratta di rendere sempre più nette le distinzioni tra le classi sociali e di riabilitare in tal modo l'opposizione della lotta di classe attraverso la quale gli operai possano diventare produttori liberi in una officina priva di padroni:

\footnotetext{
È facile osservare che per moltissimo tempo i moderni non hanno creduto che sui lavoratori ci fosse altro da dire di quanto ne aveva detto Aristotele: si daranno loro degli ordini; si riprenderanno con dolcezza, come dei bambini; si tratteranno come strumenti passivi che non hanno bisogno di pensare. Il sindacalismo rivoluzionario sarebbe impossibile se il mondo operaio dovesse avere una tale morale da deboli; il socialismo di Stato, al contrario, si troverebbe a tutto suo agio $^{26}$.
}

Le ragioni dell'apologia della violenza - che pare assurda se non la si considera sotto il profilo politico della lotta di classe ${ }^{27}$ - vanno ricercate all'interno delle dinamiche politiche della democrazia francese nel passaggio tra il XIX e il XX secolo, allorquando un rinnovato patriottismo aveva saldato la scissione tra il proletariato e la borghesia in favore di una loro pacifica convivenza domiciliata nello Stato: «Non si tratta qui di giustificare i violenti, ma di sapere quale è nel socialismo contemporaneo il compito della violenza delle masse operaie»28. Qui va sottolineato che il concetto soreliano di violenza, strettamente associato a quello di lotta di classe, può essere adeguatamente compreso solo se collocato in opposizione a quello di pace sociale ${ }^{29}$. Si tratta, difatti, di due differenti metodiche dell'azione socialista, l'una - quella della violenza - incentrata sulla scissione dalle forze borghesi e sul rifiuto di ogni

\footnotetext{
${ }^{25} \mathrm{Su}$ questo punto si veda A. Orsucci, "Tra George Sorel e Curzio Malaparte: F. Nietzsche e il sindacalismo rivoluzionario", in L. Crescenzi, C. Gentili, A. Venturelli (a cura di), Letteratura e identità europea. Per una storia dei 'buoni europei', Studi Germanici, Roma 2018, pp. 125-127.

${ }_{26}$ G. Sorel, Riflessioni sulla violenza, cit., p. 357.

${ }^{27}$ Sul nesso tra violenza e lotta di classe si veda P. Ferrario, "Sorel e il mito della violenza (Per una rilettura delle Réflexions)", cit., pp. 65-66.

28 G. Sorel, Riflessioni sulla violenza, cit., p. 128.

${ }^{29}$ Ivi, p. 311.
} 
compromesso sociale, l'altra - ovverosia quella della pace sociale - volta a trovare un'armonia tra le classi sociali tale da assicurare la conquista della forza statale ${ }^{30}$.

Il compito della violenza risiede, dunque, nella reintroduzione della scissione nei rapporti tra il proletariato e la borghesia laddove l'utopia del socialismo parlamentare aveva insediato la metafora immorale della rivoluzione - immorale perché concepita come mantenimento e consolidamento dello Stato borghese.

La violenza dello sciopero generale rappresenta per Sorel il «grande fatto sociale» ${ }^{31}$ del tempo presente e viene esaltata come un fenomeno capace di annientare la chimera della pace sociale: «[...] è la grande battaglia napoleonica (quella che annienta definitivamente $\mathrm{i}$ vinti) che gli scioperanti sperano di veder cominciare; così si produce, attraverso la pratica degli scioperi, il concetto di una rivoluzione catastrofica» ${ }^{32}$.

Il mito sociale dello sciopero generale è quindi agganciato alla pratica della violenza dell'azione proletaria poiché interviene nelle fratture della realtà senza ricomporle nell'astrazione logica dell'armonia tra le classi sociali. La moralità della violenza consiste, dunque, nel rigettare le logiche della diplomazia socialista, ovverosia le dinamiche di compromesso tra il principio della lotta di classe e l'acquisizione - di natura borghese - della forza statale: «[...] la violenza proletaria entra in scena allo stesso tempo in cui la pace sociale pretende di addolcire i conflitti; la violenza proletaria conferma i datori di lavoro nel loro ruolo di produttori e tende a restaurare la struttura

\footnotetext{
30 Per un approfondimento del concetto soreliano di violenza si veda G. Cavallari, "La genesi del concetto di violenza in Sorel", Giornale Critico della Filosofia Italiana, LIII, 1974, pp. 271303.

31 G. Sorel, Riflessioni sulla violenza, cit., p. 153: «Si approvi o si condanni quello che si chiama il metodo diretto e rivoluzionario, è evidente che esso non è prossimo a scomparire. [...] esso è il grande fatto sociale dell'ora attuale e bisogna cercare di comprenderne la portata».

${ }^{32}$ Ivi, p. 154.
} 
delle classi nella misura in cui queste sembrano mescolarsi in un pantano democratico» ${ }^{33}$.

La metodica messa in campo dal sindacalismo rivoluzionario promosso dalla cosiddetta nuova scuola soreliana è quindi una metodica della scissione volta ad esaltare le tensioni sociali e a demolire, in senso definitivo e catastrofico, il socialismo parlamentare e lo Stato in quanto sede immorale dei compromessi tra classi sociali. L’azione proletaria deve presentarsi, quindi, come un'«opera grave, spaventosa e sublime» ${ }^{34}$ da svolgersi in senso antistatalista perché indirizzata a scardinare il culto dello Stato al quale persino i socialisti sacrificano le proprie vittime: «I sindacalisti non si propongono di riformare lo Stato [...] essi vogliono distruggerlo» ${ }^{35}$.

La messa in rilievo delle opposizioni e, quindi, lo scioglimento del dramma sociale in senso catastrofico sono le componenti fondamentali del mito dello sciopero generale al quale Sorel oppone l'inadeguatezza e l'immoralità del logos. Il linguaggio rappresenta, difatti, uno strumento di mediazione tra le parti in conflitto e di astrazione dal dato immediato. In tal senso, esso è espressione dell'azione politica parlamentare volta a realizzare utopicamente la pace sociale. Al contrario, l'immagine offerta dal mito consente «di evocare in blocco e per mezzo della sola intuizione [...] la massa dei sentimenti» ${ }^{36}$ grazie alla quale i proletari si dispongono a rivoluzionare la realtà nella direzione di una nuova civiltà. Il mito diventa, in quest'ottica, un mezzo per agire sul presente:

[...] lo sciopero generale è proprio ciò che ho detto: il mito nel quale si racchiude tutto intero il socialismo, cioè a dire una organizzazione di immagini capaci di evocare istintivamente tutti i sentimenti che corrispondono alle diverse manifestazioni della guerra intrapresa dal socialismo contro la società moderna. Gli scioperi hanno fatto nascere nel proletariato i sentimenti più nobili, più profondi e più stimolanti all'azione che esso possiede; lo sciopero generale li raggruppa tutti in un quadro di insieme e, con il loro accostamento, dona a

\footnotetext{
${ }^{33}$ Ivi, p. 172.

34 Ivi, p. 234.

35 Ivi, p. 206.

${ }^{36}$ Ivi, p. 213.
} 
ciascuno di essi la sua massima intensità. [...] Otteniamo in tal modo quella intuizione del socialismo che il linguaggio non è in grado di rendere in maniera perfettamente chiara, e otteniamo ciò in un insieme che è percepito all'istante ${ }^{37}$.

La funzione del mito nella pratica dello sciopero generale sindacalista è quindi quella di rimarcare adeguatamente le differenze tra le classi sociali e di consentire il ristabilimento del campo di battaglia entro cui potrà consumarsi l'ultima decisiva disfatta della borghesia e dello Stato moderno. In quest'ottica, soltanto per mezzo del mito dello sciopero generale sarà possibile azionare il principio della lotta di classe e, con esso, la forza motrice della classe operaia.

Nel mito dello sciopero generale Sorel racchiude, dunque, il passaggio dal capitalismo al socialismo descrivendolo come una catastrofe irrappresentabile coi soli mezzi del logos. Esso è una perfetta rappresentazione del movimento proletario nei suoi periodi di azione, e cioè nel movimento creatore che interviene a plasmare una nuova civiltà nei rari momenti di libertà della coscienza.

\section{Sciopero generale sindacalista $v s$ sciopero generale politico}

Il grande fatto sociale rappresentato dallo sciopero generale, al quale Sorel dedica un'attenta e minuziosa analisi, introduce la necessità di operare delle distinzioni. Nello specifico, il fondatore della cosiddetta nuova scuola si sofferma ad individuare le differenze che intercorrono tra lo sciopero generale sindacalista e lo sciopero generale politico con lo scopo di collocare il primo in una zona di opposizione rispetto alla dimensione politico-statale.

Lo sciopero generale sindacalista, come si è detto nel paragrafo precedente, rappresenta un tutto indivisibile e, cioè, un'azione capace di rappresentare la totalità del socialismo proletario, promuovendo un'istanza di rinnovamento morale perché non individualisticamente concepita.

${ }^{37}$ Ivi, pp. 219-220. 
Lo sciopero generale politico si colloca, invece, all'interno della prospettiva utilitaristica e statalista poiché configura un mezzo di cui i politicanti si servono per avvicendarsi al potere statale: «[...] in tali condizioni il sollevamento popolare deve avere per fine il passaggio del potere da un gruppo di politicanti a un altro gruppo di politicanti, il popolo rimanendo sempre la povera bestia che porta il basto» ${ }^{38}$.

Nel caso dello sciopero generale politico, la fede nello Stato con-fonde le forze sociali in campo, sciogliendo il principio della lotta sociale all'interno della comune lotta per la conquista della forza statale. In tal senso, il socialismo parlamentare opera conformemente ai dettami della civiltà borghese salvaguardandone l'assetto capitalistico. Al contrario, lo sciopero sindacalista promuove un'azione di trasformazione assoluta della realtà sociale nella direzione della distruzione dello Stato in quanto principio garante del compromesso tra le classi sociali:

\begin{abstract}
Questa gravità dell'opera perseguita dal proletariato non converrebbe certo alla gioiosa clientela dei nostri politicanti; questi vogliono rassicurare la borghesia promettendole di non lasciare che il popolo si abbandoni ai suoi istinti anarchici; le spiegano che neppure si sognano di sopprimere la grande macchina dello Stato, e che quanto i socialisti saggi desiderano sono due cose: impadronirsi di questa macchina per perfezionare gli ingranaggi e farli funzionare nel migliore interesse dei loro amici; rendere il governo più stabile, ciò che sarà di grande vantaggio per tutti gli uomini di affare ${ }^{39}$.
\end{abstract}

Nello sciopero generale sindacalista Sorel individua, dunque, l'azione dell'uomo autenticamente libero perché svincolato dalle logiche utilitaristiche interne allo Stato e gestite dai politicanti di professione. Esso si presenta come il grande motore della storia, e cioè nei termini di una guerra totale atta a sovvertire lo Stato.

Nello sciopero generale politico è implicata, invece, la nozione soreliana di forza che ne rivela il carattere precipuamente borghese. Difatti, esso implica l'avvicendamento delle classi sociali al potere statale e, dunque, il

\footnotetext{
38 Ivi, pp. 255-256.

${ }^{39}$ Ivi, pp. 262-263.
} 
rafforzamento dello Stato pur nel variare delle minoranze al suo governo. Nel caso dello sciopero sindacalista, la violenza proletaria elimina ogni ordine sociale organizzato dalle minoranze di volta in volta al potere scardinando, di fatto, il meccanismo di trasformazione sociale gestito dallo Stato e dalle dinamiche della democrazia parlamentare, e per questo finalizzato alla conservazione del capitalismo borghese.

La differenza tra lo sciopero politico e lo sciopero sindacalista ripropone, in definitiva, la critica soreliana nei riguardi del socialismo riformista ritenuto essere un imitatore delle tendenze borghesi e, quindi, incapace di attuare il passaggio dal capitalismo al socialismo. La realizzazione di tale passaggio può avvenire, nell'ottica soreliana, soltanto attraverso un lavoro sotterraneo, e cioè distante e distinto dalle dinamiche della rappresentanza parlamentare. Esso implica uno sforzo eroico poiché privo di profitti: «È veramente sottoterra che si produce il movimento sindacalista; gli uomini che vi si dedicano non fanno tanto chiasso nella società; che differenza tra essi e i vecchi capi della democrazia al lavoro per la conquista del potere!» ${ }^{40}$.

\section{Conclusioni}

Nelle Riflessioni sulla violenza Georges Sorel compie un'interessante operazione di riabilitazione delle forze irrazionali e simboliche nel contesto della teoria politica delle lotte sociali. La messa in campo del mito, inteso come costruzione immaginifica capace di rappresentare ed esasperare il conflitto tra le classi sociali, consente all'autore francese di prendere le distanze da ogni forma di socialismo di Stato, presentato come un movimento interno, e quindi conforme, alle dinamiche del capitalismo borghese. Ad accomunare socialisti riformisti e capitalismo sarebbe, nella prospettiva soreliana, il fascino esercitato dallo Stato e il culto che ne deriva. Se rimane dentro lo Stato, l'azione socialista non può schiudere alcun progetto autenticamente

${ }^{40}$ Ivi, p. 344. 
storico poiché si limita a perpetrare la subordinazione delle masse operaie agli interessi politici, sebbene nella direzione di un progressivo e graduale miglioramento. Il mito, al contrario, comporta l'evocazione simultanea e sublime dei sentimenti della massa e, quindi, un'azione violenta ed immediata capace di trasfigurare la realtà nella direzione di una civiltà rinnovata.

Nelle Riflessioni il mito sociale dello sciopero generale implica un'istanza antistatale e, dunque, anarchica, poiché in favore di una auto-organizzazione dei produttori contro le dinamiche politico-statali dei partiti. Nella ricezione del pensiero soreliano non mancano, tuttavia, esempi di applicazione del mito sociale disgiunto dalla pratica dello sciopero generale, talvolta in un'ottica reazionaria. Va però sottolineato - pur in maniera cursoria - che nelle diverse modulazioni della riflessione soreliana (per esempio, nel contesto del fascismo italiano) si riflettono, oltre alle diverse condizioni politico-sociali nelle quali essa viene introdotta, tutti i limiti di una teoria del mito che non approfondisce i contenuti programmatici dell'azione ma che tende ad esaltare innanzitutto il carattere della spontaneità e dell'irrazionale all'interno delle lotte sociali. Tale vaghezza ha comportato la possibilità di interpretare il mito come uno strumento neutro capace di adattarsi ad obiettivi sociali completamente diversi ${ }^{41}$ : «Non rimane che la volontà, la spontaneità, che può quindi essere applicata a qualsiasi contenuto» ${ }^{42}$.

\footnotetext{
${ }^{41} \mathrm{Sul}$ carattere vago del mito sociale soreliano sul piano dei contenuti programmatici si veda P. Ferrario, "Sorel e il mito della violenza (Per una rilettura delle Réflexions)", cit., p. 79: "Sorel rimane tuttavia vago ed impreciso per quel che riguarda una spiegazione dei possibili aspetti concreti che la pratica dello sciopero generale potrà rivestire».

${ }^{42}$ S. Minelli, "Mito, utopia e propaganda: linguaggio di verità o tecniche di manipolazione?", Thomas Project. A border journal for utopian thoughts, III, 2020, p. 40. Sull'interpretazione di Sorel come precursore dell'ideologia fascista cfr. M. Ashéri, Z. Sternhell, M. Sznajder, Naissance de l’idéologie fasciste, Gallimard, Paris, 1994.
} 


\section{Nota bibliografica}

\section{Testi di Georges Sorel}

SOREL, Georges, "Y-a-t-il de l'utopie dans le Marxisme?", Revue de Métaphysique et Morale, II, 7e année, 1899, pp. 152-175.

—, Lo sciopero generale e la violenza, s.e., Roma 1906.

_, "Réflexions sur la violence”, Librairie de «Pages Libres», Paris 1908.

—, Scritti politici, a cura di R. Vivarelli, UTET, Milano 2006.

\section{Letteratura secondaria citata}

ANDREU, Pierre, "Bergson et Sorel”, Les études bergsoniennes, vol. III, Albin Michel, Paris 1952, pp. 41-78.

ASCHÉRI, Maia, STERNHELL, Zeev, SZNAJDER, Mario, Naissance de l’idéologie fasciste, Gallimard, Paris 1994.

BERGSON, Henri, Essai sur les données immédiates de la conscience, Félix Alcan, Paris 1889; tr. it. di G. Bartoli, Saggio sui dati immediati della coscienza, Bollati Boringhieri, Torino 1964; tr. it. di F. Sossi, Raffaello Cortina Editore, Milano 2002.

CAVALLARI, Giovanna, "La genesi del concetto di violenza in Sorel", Giornale Critico della Filosofia Italiana, LIII, 1974, pp. 271-303.

CURTIS, Michael, Three Against the Third Republic, Sorel, Barrès, and Maurras, Princeton University Press, Princeton 1959.

FERRARIO, Paolo, "Sorel e la critica al marxismo”, Il Politico, XLVIII/3, 1983, pp. 557-574. 
—, "Sorel e il mito della violenza (Per una rilettura delle Réflexions)", Il Politico, LI/1, 1986, pp. 63-81.

GERVASONI, Marco, Georges Sorel. Una biografia intellettuale. Socialismo e liberalismo nella Francia della Belle époque, Unicopli, Milano 1997.

HUGHES, Henry Stuart, Consciousness and society: the reorientation of European social thought, 1890-1930, Knopf, New York 1958.

MINELLI, Sara, "Mito, utopia e propaganda: linguaggio di verità o tecniche di manipolazione?", Thomas Project. A border journal for utopian thoughts, III, 2020, pp. 32-47.

ORSUCCI, Andrea, "Tra George Sorel e Curzio Malaparte: F. Nietzsche e il sindacalismo rivoluzionario", in L. Crescenzi, C. Gentili, A. Venturelli (a cura di), Letteratura e identità europea. Per una storia dei 'buoni europei', Studi Germanici, Roma 2018, pp. 125-127.

SAND, Shlomo, "Bibliographie des études sur Georges Sorel", Mil neuf cent. Revue d'histoire intellectuelle, I, 1983, pp. 173-206.

SALVATORELLI, Luigi, “Georges Sorel”, Pegaso, II, 1930, pp. 28-42.

TAGER, Michael, "Myth and Politics in the Works of Sorel and Barthes", Journal of the History of Ideas, XLVII, 1986, pp. 625-639.

\section{Nota biografica}

Viviana Galletta ha conseguito la laurea magistrale in Scienze Filosofiche nel 2019 presso l'Università degli Studi di Catania e, contestualmente, il Diploma di Licenza Magistrale presso la Scuola Superiore di Catania. Attualmente è dottoranda presso l'Università degli Studi di Catania nel corso di Scienze dell'Interpretazione (XXXVI ciclo) con un progetto di ricerca dedicato all'opera filosofica di Franciscus Hemsterhuis. 